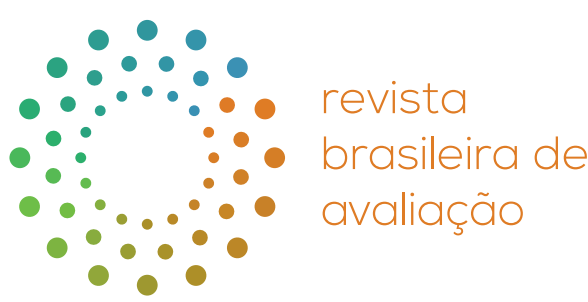

\title{
Una evaluación ‘sin' evaluadores. Participación ciudadana en la Agenda 2030
}

\section{An evaluation 'without' evaluators. Citizen participation in the 2030 Agenda}

Esteban Tapella ${ }^{1 *}[$ branco] (1)

${ }^{1}$ Universidad Nacional de San Juan (UNSJ), San Juan, Argentina

CÓMO CITAR: Tapella, Esteban (2021). Una evaluación 'sin' evaluadores. Participación ciudadana en la Agenda 2030. Revista Brasileira de Avaliação, 10(2), e101321. https://doi.org/10.4322/rbaval202110013

\section{Resumen}

Este artículo sitúa la evaluación participativa en el marco de la Agenda 2030 y los Objetivos de Desarrollo Sostenible, los que sostienen la urgente necesidad de la inclusión social y el protagonismo de la sociedad civil en la construcción de un mundo donde "nadie se quede atrás". El trabajo aboga por una evaluación inscripta en una perspectiva transformadora de la realidad, capaz de crear un diálogo reflexivo y crítico entre múltiples actores que se vinculan con una intervención, programa o proyecto. Se valora la evaluación participativa como un enfoque pertinente al actual contexto de América Latina, destacando la importancia de crear oportunidades para participar así como capacidades para desarrollar un protagonismo transformador de la ciudadanía.

Palabras clave: Evaluación. Participación social. Fortalecimiento de capacidades.

\begin{abstract}
This article places participatory evaluation within the framework of the 2030 Agenda and the Sustainable Development Goals, which support the urgent need for social inclusion and the role of civil society in the construction of a world where "no one is left behind". The work advocates for an evaluation inscribed in a transforming perspective of reality, capable of creating a reflective and critical dialogue between multiple social actors that are connected with an intervention, program or project. Participatory evaluation is valued as a pertinent approach to the current context of Latin America, highlighting the importance of creating opportunities to participate as well as capacities to develop a transforming role of citizenship.
\end{abstract}

Keywords: Evaluation. Social participation. Capacity building.

\section{A modo de introducción}

La preocupación por la evaluación de las políticas públicas ha crecido significativamente en los últimos veinte años. Esto se refleja tanto en lo referido a la producción teórica y la consiguiente reflexión metodológica como así también en el incremento de políticas nacionales de evaluación en países de todos los continentes, la creciente institucionalidad de la evaluación y el surgimiento de diversas iniciativas orientadas a profesionalizar esta práctica (Stockmann \& Meyer, 2016). La declaración del 2015 como Año Internacional de la Evaluación por parte de la Asamblea General de Nacionales Unidas (A/RES/69/237), señala esta tendencia a nivel global, a la vez que una suerte de 'alianza' sobre el tema hace confluir el interés y las acciones de los principales organismos internacionales de desarrollo, las redes regionales
RBAVAL supports efforts related to the visibility of African descent authors in scientific production. Thus, our publications request the self-declaration of color/ethnicity of the authors to make such information visible in the journal.

Recibido: Abril 16, 2021

Aceptado: Junio 28, 2021

*Autor correspondiente:

Esteban Tapella

E-mail: etapella@gmail.com

\section{(cc) BY}

Este es un artículo publicado en acceso abierto (Open Access) bajo la licencia Creative Commons Attribution, que permite su uso, distribución y reproducción en cualquier medio, sin restricciones siempre que el trabajo original sea debidamente citado. 
de evaluación (VOPEs)' ${ }^{1}$, fundaciones, diversos organismos del Estado, las organizaciones no gubernamentales y la academia, todos ellos interesados en potenciar la evaluación como instrumento para mejorar las políticas públicas y generar aprendizajes válidos para el diseño de futuras intervenciones.

Coincidiendo con el lanzamiento de los Objetivos de Desarrollo Sostenible (ODS), el escenario se presenta como un punto de inflexión en la teoría y la práctica de la evaluación, la cual busca adaptarse a los principios que inspiran el nuevo marco global de desarrollo: centrarse en las personas, no dejar a nadie atrás, responsabilidad mutua e integralidad (Naciones Unidas, 2015). En dicho contexto se apuesta a construir una nueva agenda de prioridades para la evaluación a nivel global entre 2016 y 2030. Esta busca reducir la brecha entre la comunidad de evaluadores (oferta) y la comunidad de los hacedores de políticas (demanda), incluyendo el protagonismo de la sociedad civil como un actor central en la gestión y evaluación de las políticas públicas (Griggs et al., 2017; Feinstein, 2017). La llamada Agenda 2030 para el Desarrollo Sostenible incluye un mecanismo de seguimiento y evaluación para asegurar que los ODS sean monitoreados y revisados sistemáticamente con el protagonismo de la sociedad civil, ayudando así a los países en la implementación de dicha agenda (Bamberger et al., 2017).

La Agenda 2030 para el Desarrollo Sostenible establece una visión transformadora hacia la sostenibilidad económica, social y ambiental de los Estados que la suscribieron. Es sin duda una guía y una oportunidad histórica para América Latina y el Caribe en tanto constituye la posibilidad de materializar una serie de principios, valores e iniciativas relacionadas con la concreción de los derechos humanos, dado que se enmarcan en principios como la igualdad, la no discriminación, la participación, la rendición de cuentas y la co-responsabilidad. Dos aspectos conectan de manera estrecha los ODS con la evaluación, y es justamente el componente participativo. Los 17 objetivos de la Agenda 2030 para el Desarrollo Sostenible se plasman en 169 metas conexas, de carácter integrado e indivisible, en las que la participación de la ciudadanía tiene un rol destacado. Siguiendo a Leopoldo Fidyka (2017), vale destacar algunos ejemplos. El cuarto objetivo, referido a la educación, está cruzado transversalmente por la noción de participación, como eje para garantizar que todos los alumnos adquieran los conocimientos teóricos y prácticos necesarios para promover el desarrollo sostenible. En cuanto a la igualdad de los géneros, el quinto objetivo destaca la participación plena y efectiva de las mujeres y la igualdad de oportunidades de liderazgo a todos los niveles en la esfera política, económica y pública. Por su parte, el objetivo un décimo referido a las ciudades plantea la participación como estrategia para una urbanización inclusiva y sostenible, y la capacidad para una planificación y gestión participativas, integradas y sostenibles de los asentamientos humanos en todos los países. En cuanto a la calidad institucional, el objetivo dieciséis busca garantizar la adopción de decisiones inclusivas, participativas y representativas que respondan a las necesidades a todos los niveles. Y así, es claramente visible el llamado a la participación social de los ODS, por tanto enfoques como el de la evaluación participativa adquieren aún más relevancia en este contexto.

En este artículo intento caracterizar la evaluación participativa como un enfoque coherente con el nuevo contexto global y regional, contexto que demanda un mayor involucramiento de diversos actores sociales en la agenda evaluativa. Este mayor protagonismo de la sociedad civil es visto acá como una forma de asegurar, no sólo que las evaluaciones respondan a las necesidades de las personas usuarias, sino también que se ajusten a estándares de calidad, se basen en evidencias, sean creíbles y puedan ser utilizadas para mejorar las políticas públicas (Patton, 2017; Torrejon-Cardona et al., 2017). En los próximos párrafos destacaré aquellos aspectos que hacen de la evaluación una práctica emancipadora capaz de fortalecer

\footnotetext{
1 Las Organizaciones Voluntarias para la Profesionalización de la Evaluación (VOPEs, por sus siglas en inglés) son los foros abiertos donde los evaluadores y los interesados en la evaluación se conectan, reúnen y se capacitan. Materializadas a través de redes o asociaciones de profesionales, procuran ser portadoras de valores sobre la transparencia, la responsabilidad democrática y el enfoque de derechos en la práctica evaluativa. Durante los últimos quince años ha existido un crecimiento exponencial de estas organizaciones, pasando de 19 VOPEs localizadas sólo en países desarrollados en el año 2000, a unas 170 VOPEs con presencia en todos los continentes y regiones. La Organización Internacional para la Cooperación en Evaluación (IOCE) reúne a las VOPEs regionales y nacionales, ayudando a legitimar la evaluación y apoyar estas organizaciones para que puedan contribuir mejor a la buena gobernanza, la toma de decisiones eficaces y fortalecer el papel de la sociedad civil.
} 
la democracia y construir una sociedad más justa, inclusiva y solidaria. Buena parte de estas reflexiones han surgido y se recrean constantemente en EvalParticipativa ${ }^{2}$, la comunidad de práctica y aprendizaje sobre evaluación participativa para América Latina y el Caribe.

\section{La participación ciudadana: mucho más que un simulacro simbólico}

En los últimos años han cobrado importancia diferentes enfoques de evaluación que implican un mayor protagonismo de diferentes actores sociales. Entre otros, vale destacar la Evaluación Democrática (Segone, 1998), la Evaluación de Procesos o Sistematización de Experiencias (Jara-Holliday, 2010, 2013; Tapella \& Rodríguez Bilella, 2014a, 2014b, 2014c, 2015), el Cambio Más Significativo (Davis \& Dart, 2005), el Enfoque Sistémico en Evaluación (Boyd et al., 2007), la Evaluación de la Complejidad (Rogers, 2008) y los Enfoques Colaborativos en Evaluación (Cousins et al., 2013; Cousins, 2019; Shulha et al., 2015). Estos enfoques reflejan una nueva sensibilidad en el campo de la evaluación, reconociendo la importancia que tiene el contexto y la perspectiva de los actores a la hora de valorar o atribuir efectos y resultados a una determinada intervención o generar lecciones aprendidas a partir de ella (Patton, 2010, 2017). De forma simultánea, el discurso público ha enfatizado recurrentemente la necesidad de fortalecer el protagonismo de la sociedad civil, destacando la dimensión participativa como un aspecto central de la práctica evaluativa (Jacob et al., 2009). Conceptos tales como 'participación', 'acompañamiento', 'perspectiva de los actores', entre otras, resultan cada vez más frecuentes en el campo evaluativo (Jacob \& Ouvrard, 2009; Jacob et al., 2009).

Sin embargo, más allá del discurso y las buenas intenciones, la práctica de la evaluación no siempre ha reflejado esta 'vocación' participativa (Guijt, 2014), dado que la noción de participación adquiere diferentes connotaciones. Muchas veces los programas y proyectos tendientes a 'estimular' la participación ignoran que cualquier ejercicio de participación puede convertirse en un simulacro simbólico, si es que no se toma conciencia que animar un proceso participativo implica una práctica de redistribución del poder (Chambers, 2003). De ese modo, vemos con frecuencia que evaluaciones 'participativas' se limitan a una mera instancia de consulta (participación pasiva), sin ofrecer a los actores locales la posibilidad de influir en las decisiones respecto de la agenda evaluativa. Esta serie de prácticas recurrentes evidencia cierta falta de claridad teórica y pobreza instrumental en buena parte de las evaluaciones, sin que las razones de este déficit hayan sido claramente identificadas.

Un claro ejemplo de esto se evidenció en el proceso de selección de casos para participar del primer Encuentro de Experiencias de Evaluación Participativa de América Latina y el Caribe, realizado por EvalParticipativa en Quito, Ecuador (noviembre de 2019). La convocatoria, de alcance regional, solicitó la postulación de casos y experiencias de evaluación participativa que cumplieran los siguientes principios: (a) participación de diferentes actores implicados, trascendiendo la mera acción consultiva hacia los beneficiarios y otros actores locales, y apuntando a que crezca su rol activo y decisorio en las distintas fases de la evaluación; (b) aprendizaje, como mecanismo capaz de construir capacidades locales y apoyar el fortalecimiento institucional en aras de la transformación de la realidad y el logro de un desarrollo más inclusivo; y (c) flexibilidad, como una modalidad que privilegia el contexto donde se desarrolla la evaluación por sobre cualquier acercamiento metodológico o procedimiento existente, brindando especial atención a la adecuación socio-cultural de los mismos. Se presentaron treinta experiencias, de los cuales sólo catorce cumplían parcialmente estos criterios. Si bien la mayoría de los casos se autodenominaba 'participativa', las instancias de participación mencionadas se limitaban a un involucramiento pasivo de informantes claves. Apenas ocho, de los treinta casos, involucraba a los actores en la definición de los temas y preguntas de evaluación, la selección de los instrumentos y la recolección de datos; mientras

\footnotetext{
${ }^{2}$ EvalParticipativa es una iniciativa conjunta entre el Programa de Estudios del Trabajo, el Ambiente y la Sociedad (PETAS) de la Universidad Nacional de San Juan (Argentina) y el proyecto Focelac para el fomento de capacidades y articulación de actores de la evaluación en América Latina del Instituto Alemán de Evaluación para la Cooperación al Desarrollo (DEval). A través de la creación de una Comunidad de Práctica y Aprendizaje en Evaluación Participativa (EP) para América Latina y el Caribe, el proyecto busca contribuir con el fortalecimiento e involucramiento inclusivo de la sociedad civil en procesos evaluativos de la región. Más información en EvalParticipativa (2021).
} 
que ninguno de ellos involucró activamente a los participantes en el análisis de los datos y la elaboración de las conclusiones y recomendaciones. Sólo cuatro del total apoyaron una participación activa en la socialización de los resultados de la evaluación.

¿Cómo lograr entonces la participación y el protagonismo de diversos actores sociales en la evaluación de los programas o proyectos que los afectan o involucran? La respuesta pasa por identificar las condiciones y los mecanismos que hacen posible una efectiva participación ciudadana en los procesos de evaluación, sin que ello menoscabe la calidad y rigor exigibles a las evaluaciones (Rodríguez-Bilella et al., 2016). Es necesario profundizar en experiencias evaluativas que permitan entender los aspectos que hacen la diferencia en una evaluación en términos de participación social real y efectiva, explorando los posibles aportes de los enfoques participativos y colaborativos a las evaluaciones convencionales (Rodríguez-Bilella y Tapella, 2018).

\section{¡Déjame hacerlo, enséñame a hacerlo!}

La noción de participación, según mi punto de vista, surge a partir del encuentro de dos dinámicas: la oportunidad de participary la capacidad de participar. La primera está determinada por la voluntad institucional y política de quien diseña y conduce una evaluación por crear espacios reales de participación. La segunda está determinada principalmente por las actitudes y habilidades que los actores de la sociedad civil han desarrollado a través de experiencias anteriores. Es posible hablar de participación cuando existe una adecuación entre ambas dinámicas; es decir, las capacidades de participar de los pobladores se ajustan a las oportunidades que 'abre' la institución, el programa o el proyecto (Tapella \& Sanz, 2019).

En cuanto a la oportunidad de participar, la experiencia me indica que no todas las instituciones/programas que convocan a una evaluación participativa están realmente dispuestas a facilitar y acompañar estos procesos (Sanz et al., 2019). Entre los principales retos, me gustaría destacar aquellos que siento más relevantes:

- es importante que los representantes de los diferentes niveles del programa evaluado tengan real disposición para una evaluación de este tipo, que sean conscientes de la implicancia que una evaluación multi-actoral y desde las bases puede tener, y estén abiertos a escuchar y adoptar las recomendaciones que de ella puedan surgir;

- la institución/programa debe contar con tiempo y recursos, ya que crear verdaderos espacios para el protagonismo de la sociedad civil implica procesos lentos, largos y costosos, y no siempre las instituciones están dispuestas a asumirlos;

- es muy importante contar con el apoyo de un facilitador/a local con conocimiento de la realidad e idiosincrasia de los participantes, así como adecuado manejo de herramientas de comunicación y animación socio-cultural;

- es determinante que la institución solicite o genere instancias de capacitación para los actores locales involucrados, ya que en la mayoría de los casos la experiencia participativa y evaluativa de los principales interesados es muy limitada; y

- es muy importante conformar un equipo de evaluación relativamente pequeño pero integrado con representantes de todas las partes involucradas, el que deberá actuar durante todo el proceso, desde la priorización de los temas más relevantes y la formulación de las preguntas y objetivos, hasta la comunicación y facilitación del uso de los resultados, pasando por el trabajo de definición de indicadores, recopilación y análisis de los datos así como elaboración de conclusiones y recomendaciones.

En cuanto a la capacidad de participar, siento que quienes apoyamos estos procesos tenemos un gran desafío. Particularmente en contextos como el latinoamericano, por mucho tiempo rígido y reticente a crear experiencias verdaderamente democráticas y participativas. Sin duda, las capacidades de participación se crean cuando desde una institución se ofrecen instancias de participación y se anima a los pobladores y otros actores a involucrarse decididamente en la práctica evaluativa. Pero, sumado a la existencia de 'oportunidades de participación', las que funcionan como una motivación fundamental para los participantes, es necesario 
iniciar un proceso fortalecimiento de capacidades continuo (Tapella \& Sanz, 2019). Según mi experiencia, los principales desafíos son los siguientes:

- adecuar las instancias de participación a las capacidades de los actores, buscando intereses comunes y al alcance de los participantes (por ejemplo, no pretender que los miembros de una organización de base se involucren en el diseño de un muestreo con representación estadística para una evaluación de impacto) y acompañando los desafíos que ellos estén dispuestos a asumir;

- conocer el variado conjunto de herramientas para la evaluación democrática y participativa que hoy existe y estar dispuestos a adecuarlas y/o recrearlas para que sirvan a los procesos participativos que requiere la evaluación (elaboración de herramientas apropiadas, juegos o dinámicas que garanticen el protagonismo de todos y no sólo el de los más extrovertidos, etc.);

- reconocer que las herramientas 'no lo pueden todo', es necesario evidenciar en el encuentro y diálogo con los participantes una vocación participativa, la cual se expresa en la tolerancia al error, la voluntad para 'volver a explicar', la disposición a revisar acuerdos y rediseñar el plan de trabajo, etc.; $y$

- tener claridad sobre nuestro papel, ya no de 'evaluadores' sino de 'facilitadores' de todo el proceso; y esto implica aprender a motivar sin empujar, reflexionar con el grupo sin condicionar conclusiones, aportar ideas sin imponer las propias y hacer preguntas sin sugerir las respuestas.

En base a mi experiencia personal y el análisis de diversos casos y experiencias, considero que estos son los aspectos que contribuirán con una verdadera y sincera evaluación participativa. Esto obliga a desarrollar una metodología donde las partes implicadas no-evaluadoras, es decir las personas interesadas y a quienes concierne la evaluación, especialmente gestores locales y personas beneficiarias de un programa, se involucran significativamente en el proceso de realización de la evaluación. Se trata de una evaluación 'sin' evaluadores convencionales, en la que los representantes de entidades y los beneficiarios trabajan conjuntamente en el diseño, implementación e interpretación de los datos, así como en la difusión y uso de los resultados y recomendaciones.

\section{Hacia una evaluación 'sin' evaluadores}

Cuando pensamos qué es una evaluación participativa y cuál es su implicancia para la sociedad civil, surge un sinfín de definiciones posibles, algunas más ambiciosas que otras. Me gusta pensar que una evaluación participativa es aquella donde la figura del evaluador o evaluadora convencional se desdibuja. Me imagino una evaluación 'sin' evaluadores, donde los ahora agentes externos favorecen la incorporación activa y consiente de los miembros de la organización en el proceso evaluativo. Una evaluación donde las partes involucradas definen qué será evaluado, quiénes participarán, cuándo se hará, qué métodos de recolección y análisis de datos se usará y cómo se comunicarán los resultados.

Una evaluación participativa así entendida implica un cierto nivel de control sobre las decisiones respecto de la dirección de la evaluación. Así, las partes involucradas ya no se limitan a ser fuentes de información o simples observadoras, sino que adquieren protagonismo durante todo el proceso. Las evaluaciones realizadas bajo este enfoque se desarrollan bajo el liderazgo de grupos que conocen muy de cerca el programa que se evalúa, especialmente el personal técnico que presta los servicios a nivel local y a la comunidad usuaria de esos servicios (Sanz et al., 2019).

Esta concepción diferente de evaluación no sólo servirá para tomar medidas correctivas y así obtener mejores resultados en la intervención evaluada. Tampoco se limitará, como corolario de la misma, a añadir o suprimir actividades o a cambiar la estrategia de intervención. Una evaluación con la activa participación de la sociedad civil servirá, sobre todo, para dar protagonismo y trasladar a la sociedad civil el poder de decisión. Una evaluación participativa permitirá fortalecer las organizaciones sociales para que tengan mayor control sobre su propio desarrollo, mejorando su capacidad para reflexionar, analizar y proponer soluciones desde 
sus múltiples miradas. Bajo esta mirada, una evaluación participativa será un instrumento al servicio de la democracia en nuestra región.

En el Primer Encuentro de Evaluación participativa en América Latina y el Caribe, realizado en Ecuador a finales de 2019, nos propusimos construir en conjunto con los participantes una lista de principios que podrían orientar la práctica. El resultado de aquella instancia fue muy iluminador. Personalmente siento que aquellas ideas reflejan el espíritu de una evaluación 'sin' evaluadores, donde los agentes externos adquieren un nuevo rol: facilitar procesos democráticos, reflexivos y críticos que permitan construir mejores y más inclusivas intervenciones. Una evaluación basada en el protagonismo de la sociedad civil, como lo hemos plasmado en nuestro reciente libro "Siembra y Cosecha. Manual de Evaluación Participativa" (Tapella et al., 2021), debiera:

- incorporar a los actores relevantes de la intervención o situación a evaluar de manera activa y consciente en el proceso evaluativo, en tanto sujetos de derecho;

- reconocer el valor de la experiencia de diversos actores y los conocimientos y percepción de la población local involucrada;

- crear espacios donde los representantes institucionales trabajan de modo asociado con los actores locales en el diseño, recolección, análisis e interpretación de los datos así como el uso de la información por parte de los diversos actores involucrados;

- proporcionar procedimientos, herramientas y metodologías 'amigables' que facilitan la participación de los múltiples actores, quienes suelen tener capacidades y destrezas diferentes, adoptando la técnicas y materiales didácticos para facilitar el diálogo y la construcción de consensos;

- favorecer la apropian tanto de los procesos como de los resultados de la evaluación entre los actores sociales participantes o partes interesadas; y

- contar con técnicos o profesionales que actúen como facilitadores del proceso, animadores socio-culturales que ayuden a los actores locales a diseñar la evaluación, recopilar y analizar la información, generar recomendaciones y comunicar los resultados, desarrollando las potencialidades locales y resguardando el rigor técnico de la práctica evaluativa.

Una evaluación participativa así entendida seguramente contribuirá con la construcción de una cultura de la evaluación y la institucionalización de esta práctica. Al mismo tiempo permitirá poner este instrumento al servicio de las organizaciones de la sociedad civil, que demandan y ameritan nuevos canales de participación activa e influyente en la agenda pública, y plantean a la comunidad evaluadora una seria e innovadora propuesta para mejorar nuestra práctica profesional y hacer frente a nuestro compromiso con la Agenda 2030.

\section{Fuente de financiamiento}

No hay.

\section{Conflicto de intereses}

No hay.

\section{Referencias}

Bamberger, Michael, Segone, Marco, \& Tateossian, Florencia. (2017). Evaluando los objetivos de desarrollo sostenible, con el enfoque "Que Nadie se Quede Atrás" a través de evaluaciones centradas en la equidad y con perspectiva de género. New York, USA: UN Women, IOCE y EvalGender.

Boyd, Alan, Geerling, Ted, Gregory, Wendy, Kagan, Carolyn, Midgley, Gerald, Murray, Peter, \& Walsh, Mike. (2007). Systemic evaluation: A participative, multi-method approach. The Journal of the Operational Research Society, 58(10), 1306-1320. http://dx.doi.org/10.1057/palgrave.jors.2602281

Chambers, Robert. (2003). Whose reality counts? Putting the first last (revisited ed.) London: Intermediate Technology. 
Cousins, Bradley, Whitmore, Elizabeth, \& Shulha, Lyn. (2013). Arguments for a common set of principles for collaborative inquiry in evaluation. The American Journal of Evaluation, 34(1), 7-22. http://dx.doi. org/10.1177/1098214012464037

Cousins, Bradley. (2019). Collaborative approaches to evaluation: Principles in use. Thousand Oaks: Sage.

Davis, Rick, \& Dart, Jess. (2005) The 'Most Significant Change' (MSC) tool: A guide to its use. United Kingdom: Care International, Oxfam, Ibis and Lutheran World Relief.

EvalParticipativa. (2021, julio 6). La ruta actual de EvalParticipativa: planes, acciones y sueños compartidos. Recuperado el 16 de abril de 2021, de https://evalparticipativa.net/

Feinstein, Osvaldo Nestor. (2017). Trends in development evaluation and implications for knowledge management. Knowledge Management for Development Journal, 13(1), 31-38. Recuperado el 16 de abril de 2021, de http://journal.km4dev.org/

Fidyka, Leopoldo. (2017). Los ODS como oportunidad de fortalecimiento de la participación ciudadana en el ámbito municipal. In Ponencia presentada en el XII Congreso Iberoamericano de Municipalistas, Manizales, Colombia.

Griggs, David, Nilsson, Måns, Stevance, Anne-Sophie, \& McCollum, David. (Eds.). (2017). A guide to sustainable development goals interactions: From science to implementation. Paris: International Council for Science (ICSU).

Guijt, Irene. (2014). Participatory approaches (Methodological Briefs: Impact Evaluation, No. 5). Florence, Italy: UNICEF Office of Research.

Jacob, Steve, \& Ouvrard, Laurence. (2009). Comprendre et entreprendre une évaluation participative: Guide de synthèse. Québec: Bibliothèque et Archives Nationales du Québec.

Jacob, Steve, Diallo, Nouhoun, \& Daigneault, Pierre-Marc. (2009). L'évaluation participative des politiques de coopération au développement. Analyse comparée des dispositifs du Canada, de l'Union européenne et de la Banque mondiale. Organisations \& Territoires, 18(3), 69-84. http://dx.doi.org/10.1522/revueot. v18n3.409

Jara-Holliday, Oscar. (2010). Trayectos y búsquedas de la sistematización de experiencias en América Latina. San José, Costa Rica: Centro de Estudios y Publicaciones Alforja.

Jara-Holliday, Oscar. (2013). La sistematización de las experiencias: Práctica y teoría para otros mundos posibles. Montevideo, Uruguay: Editorial EPPAL.

Naciones Unidas. (2015, octubre 21). Resolución 70/1 de la Asamblea General "Transformar nuestro mundo: la Agenda 2030 para el Desarrollo Sostenible" (A/RES/70/1). Recuperado el 16 de abril de 2021, de http://undocs.org/es/A/RES/70/1

Patton, Michael Quinn. (2010). Developmental evaluation: Applying complexity concepts to enhance innovation and use. New York, NY: Guilford Press.

Patton, Michael Quinn. (2017). Facilitating evaluation: Principles in practice. USA: SAGE Publications.

Rodríguez-Bilella, Pablo, \& Tapella, Esteban. (Eds.). (2018). Dejar huella: Historias de evaluaciones que marcaron la diferencia. Buenos Aires: Editorial UNSJ.

Rodríguez-Bilella, Pablo, Tapella, Esteban, Martinic, Sergio, Soberón, Luis, Klier, Sarah, \& Guzmán, Ana Luisa. (2016) Estándares de Evaluación para América Latina y el Caribe. Buenos Aires: Editorial Akian.

Rogers, Patricia. (2008). Using programme theory to evaluate complicated and complex aspects of interventions. Evaluation, 14(1), 29-48. http://dx.doi.org/10.1177/1356389007084674

Sanz, Juan Carlos, Tapella, Esteban, \& Meneses, Andrea. (2019). Participatory evaluation of cancer prevention and care services: a case study from Valle de la Estrella, Costa Rica. In Bradley Cousins (Ed.), Collaborative approaches to evaluation: Principles in use (Chap. II, pp. 47-53). Thousand Oaks: SAGE Publications.

Segone, Marco. (1998). Evaluación democrática (Documento de Trabajo, No. 3). Colombia: Oficina Regional para América Latina y El Caribe, UNICEF.

Shulha, Lyn, Withmore, Elizabeth, Cousins, Bradley, Gilbert, Nathalie, \& Al Hudib, Hind. (2015). Evidence based principles to guide collaborative approaches to evaluation: Technical report (Trabajo inédito) Ottawa: Centre for Research on Educational and Community Services, University of Ottawa.

Stockmann, Reinhard, \& Meyer, Wolfgang. (2016). The future of evaluation: Global trends, new challenges, shared perspectives. Switzerland: Springer. http://dx.doi.org/10.1057/9781137376374_1.

Tapella, Esteban, \& Rodríguez-Bilella, Pablo. (2014a). Shared learning and participatory evaluation. The sistematización approach to assess development interventions. Evaluation, 20(1), 115-133. http://dx.doi. org/10.1177/1356389013516055

Tapella, Esteban, \& Rodríguez-Bilella, Pablo. (2014b). Sistematización: What's that? Introducing a multistakeholder, reflexive action-learning evaluation approach. Evaluation Connections, 13-14

Tapella, Esteban, \& Rodríguez-Bilella, Pablo. (2014c). Evaluación y aprendizaje desde la práctica: La sistematización de experiencias. Knowledge Management for Development, 10(1), 52-65. Recuperado el 16 de abril de 2021, de http://www.km4dev.org/

Tapella, Esteban, \& Rodríguez-Bilella, Pablo. (2015) “Evaluaciones para aprender, aprendizajes para mejorar: Sistematización de un proyecto de desarrollo sustentable en Argentina", en Nerio Neirotti, María 
Eugenia Brisson \& Matías Matallini (Eds.), La evaluación de las políticas públicas: Reflexiones y experiencias en el escenario actual de transformaciones del Estado (Colección Políticas Públicas, pp. 135-150). Buenos Aires: Ediciones UNLa y REUN.

Tapella, Esteban, \& Sanz, Juan Carlos. (2019). La otra mirada: Evaluación participativa y mejora de los Servicios de Prevención y Atención del Cáncer en Valle de la Estrella, Costa Rica. Gestión y Análisis de Políticas Públicas. Nueva Época, (22), 102-119. http://dx.doi.org/10.24965/gapp.i22.10593

Tapella, Esteban, Rodríguez-Bilella, Pablo, Sanz, Juan Carlos, Chavez Tafur, Jorge, \& Espinosa Fajardo, Julia. (2021). Siembra y cosecha: Manual de evaluación participativa. Bonn, Alemania: Deval.

Torrejon-Cardona, Eryka, Noboa, Alejandro, \& Oraisón, Mercedes. (2017). La participación en América latina: Formas emergentes y cristalizadas de politicidad y prácticas ciudadanas. De Prácticas y Discursos, 6(7), 1-9. 